\title{
Functional correlates of optic flow motion processing in Parkinson's disease
}

\section{Deepti Putcha ${ }^{1,2}$ *, Robert S. Ross ${ }^{1,2,3}$, Maya L. Rosen ${ }^{1,2}$, Daniel J. Norton ${ }^{1}$, Alice Cronin-Golomb ${ }^{1}$, David C. Somers ${ }^{1,2}$ and Chantal E. Stern ${ }^{1,2}$}

1 Department of Psychology, Center for Memory and Brain, Boston University, Boston, MA, USA

${ }^{2}$ Athinoula A. Martinos Center for Biomedical Imaging, Massachusetts General Hospital, Boston, MA, USA

${ }^{3}$ Department of Psychology, University of New Hampshire, Durham, NH, USA

\section{Edited by:}

Sharon Gilaie-Dotan, UCL, UK

Reviewed by:

Patrizia Fattori, University of

Bologna, Italy

N. Andrew Browning, Boston

University, USA

*Correspondence:

Deepti Putcha, Department of Psychology, Center for Memory and

Brain, Boston University, 2

Cummington Mall, Boston, MA

02215, USA

e-mail:dputcha@bu.edu
The visual input created by the relative motion between an individual and the environment, also called optic flow, influences the sense of self-motion, postural orientation, veering of gait, and visuospatial cognition. An optic flow network comprising visual motion areas V6, $\mathrm{V} 3 \mathrm{~A}$, and $\mathrm{MT+}$, as well as visuo-vestibular areas including posterior insula vestibular cortex (PIVC) and cingulate sulcus visual area (CSv), has been described as uniquely selective for parsing egomotion depth cues in humans. Individuals with Parkinson's disease (PD) have known behavioral deficits in optic flow perception and visuospatial cognition compared to age- and education-matched control adults (MC). The present study used functional magnetic resonance imaging (fMRI) to investigate neural correlates related to impaired optic flow perception in PD. We conducted fMRI on 40 non-demented participants (23 PD and $17 \mathrm{MC}$ ) during passive viewing of simulated optic flow motion and random motion. We hypothesized that compared to the MC group, PD participants would show abnormal neural activity in regions comprising this optic flow network. MC participants showed robust activation across all regions in the optic flow network, consistent with studies in young adults, suggesting intact optic flow perception at the neural level in healthy aging. PD participants showed diminished activity compared to MC particularly within visual motion area MT+ and the visuo-vestibular region CSv. Further, activation in visuo-vestibular region CSv was associated with disease severity. These findings suggest that behavioral reports of impaired optic flow perception and visuospatial performance may be a result of impaired neural processing within visual motion and visuo-vestibular regions in PD.

Keywords: Parkinson's disease, fMRI, optic flow, CSv, MT+

\section{INTRODUCTION}

Parkinson's disease (PD) is a progressive neurodegenerative disorder characterized by tremor, rigidity, postural instability, bradykinesia, and gait disturbance (Young et al., 2010), as well as non-motor dysfunction including perceptual deficits (Davidsdottir et al., 2008; Armstrong, 2011) and visuospatial impairment (Amick et al., 2006; Stepkina et al., 2010; Poletti et al., 2012). Some of the visuospatial impairments seen in PD patients may be related to changes in how egocentric visual motion, or optic flow, information is processed. Identifying changes in the neural basis of optic flow processing in early PD may provide insight into how visuospatial cognition becomes dysfunctional in this disorder.

Optic flow perception has been linked to disturbances in gait (Davidsdottir et al., 2008), heading direction (Chou et al., 2009) and navigational abilities (Young et al., 2010) in PD, and is a critical aspect of visuospatial cognition (Warren et al., 2001). Optic flow paradigms mimic flow field motion as it is experienced in everyday life and include important visual information about our own movement (egomotion) as well as the environment we are moving through (Durant and Zanker, 2012). Prior functional magnetic resonance imaging (fMRI) studies have identified a network of human cortical areas that are responsive to optic flow motion processing, comprising visual motion and visuovestibular integration regions. Visual motion areas include the MT complex (MT+; Tootell et al., 1997; Seiffert et al., 2003; Duffy, 2009), area V6, located in the dorsal parieto-occipital sulcus (Pitzalis et al., 2006), and area V3A, which is positioned laterally and inferior to the parieto-occipital sulcus (Tootell et al., 1997; Cardin and Smith, 2010). The optic flow network also includes vestibular regions thought to process visual input, including the parieto-insular vestibular cortex (PIVC) and cingulate sulcus visual area (CSv; Wall and Smith, 2008; Cardin and Smith, 2010). PD pathology impacts optic flow perception at the behavioral level, characterized by veering and navigational difficulty (Davidsdottir et al., 2008; Young et al., 2010), and structurally, as observed in atrophy of regions of the parietal lobe and parietooccipital sulcus (Tinaz et al., 2011).

We used fMRI to investigate neural correlates of optic flow processing with a focus on previously delineated optic flow 
processing areas of the brain in PD patients and age-matched healthy control participants (MC). Controlling for local motion energy, we contrasted activation responses during coherent and egocentric flow field visual motion processing from noncoherent, random motion processing, in order to isolate effects from optic flow. Additionally, we examined the ability of participants to determine the direction of coherent motion, and related these findings to PD severity.

\section{MATERIALS AND METHODS PARTICIPANTS}

Twenty-four individuals diagnosed with PD and 20 healthy MC adults were initially enrolled. One MC participant was excluded because neuro-ophthalmological examination revealed advanced glaucoma. Two additional MC participants and one PD participant were excluded on the basis of excess motion in the scanner, resulting in a total of $17 \mathrm{MC}$ (7 men, mean age 62.1 years, 1 lefthanded) and 23 PD (12 men, mean age 63.5 years, 3 left-handed). All participants provided informed consent in a manner approved by the institutional review boards at Boston University and Massachusetts General Hospital. All study procedures conformed to ethical standards of experiments with human subjects. PD and MC were matched on age, education, male-to-female ratio and visual acuity, and were screened for neurological and psychiatric illness (Table 1).

Participants diagnosed with idiopathic $\mathrm{PD}$ were recruited from the PD Center at Boston Medical Center. All participants taking anti-parkinsonian medications were scanned at peak "ON" levels of medication, and all neurocognitive testing was done when participants were on medication. All patients were on a combination of levodopa-carbidopa, dopamine receptor agonists, or monoamine oxidase B inhibitors. Four patients were also on antidepressant medication, and two other patients were on anti-anxiety medication as needed. Levodopa Equivalent Dosage was calculated as per convention (Tomlinson et al., 2010) to be $410.61 \mathrm{mg} /$ day on average in the PD group. Individuals met the clinical criteria for mild to moderate PD (Hoehn and Yahr stages

Table 1 | Participant demographics, perceptual screening, and mood assessment.

\begin{tabular}{lcc}
\hline & MC (N= 17) & PD (N= 23) \\
\hline Age (years) & $62.1 \pm 9.3$ & $63.5 \pm 5.9$ \\
Male/Female & $7 / 10$ & $12 / 11$ \\
Education (years) & $16.9 \pm 2.2$ & $17.7 \pm 2.1$ \\
UPDRS Total & - & $26.8 \pm 11.5$ \\
UPDRS Motor & - & $15.6 \pm 7.5$ \\
Levodopa Equivalent Dosage (mg/day) & - & $410.6 \pm 263.6$ \\
Hoehn and Yahr & - & 2 (median) \\
Visual Acuity Near (log transform) & $0.06 \pm 0.12$ & $0.03 \pm 0.27$ \\
Visual Acuity Far (log transform) & $0.01 \pm 0.11$ & $-0.08 \pm 0.23$ \\
Beck Depression Inventory* & $2.2 \pm 3.1$ & $6.1 \pm 4.7$ \\
Beck Anxiety Inventory** & $1.9 \pm 2.1$ & $5.8 \pm 3.8$ \\
\hline
\end{tabular}

Values presented in the table are means \pm standard deviations, unless otherwise noted. UPDRS: Unified Parkinson's Disease Rating Scale. ${ }^{*} p<0.01$, ${ }^{*} p<0.001$.
I-III) as assessed by the Unified Parkinson's Disease Rating Scale (UPDRS; Movement Disorder Society Task Force on Rating Scales for Parkinson's Disease, 2003). The median Hoehn and Yahr staging was 2.0, ranging from 1.0 (unilateral) to 3.0 (moderate bilateral). Average total score on the UPDRS was 26.8 and average motor sub-score was 15.5.

All participants were screened for contraindications to MRI. At study entry, the Mini-Mental State Examination (MMSE) was administered to screen for mental status and rule out dementia; no participant met criteria for dementia (Table 1). All participants received detailed health history screening and a neuroophthalmological examination to ensure eye integrity and rule out ocular disease or abnormality. Other exclusionary criteria included coexisting, chronic medical illnesses, use of psychoactive medication besides antidepressants and anxiolytics in the MC group (allowed in PD), history of intracranial surgery or head trauma resulting in a loss of consciousness, and history of drug and alcohol abuse.

\section{NEUROIMAGING PROCEDURE}

Each scanning session included $20 \mathrm{~min}$ of structural imaging sequences followed by six runs of the functional optic flow. Each functional run (duration: $4 \min 24 \mathrm{~s}$; repetition time $=2 \mathrm{~s}$ ) consisted of 8 cycles of 16-second alternating blocks of flow motion ("flow") and random motion ("random"), with the first condition randomly chosen. 2000 moving white dots (each 2 arcmin $\times 2$ arc-min; dot duration $500 \mathrm{~ms}$ ) were presented within a full screen aperture measuring $10.5^{\circ}$ (height) by $16.7^{\circ}$ (width). Dot density was 4.14 dots $/ \mathrm{cm}^{2}$. In both the "flow" and "random" conditions, dot speed scaled with radial distance from the fixation cross (Figure 1). In the "flow" condition, all dots moved with a coherent expansion/contraction direction and/or consistent rotation direction about the central fixation cross (focus of expansion/contraction). In the "random" condition, the dot speed was the same as in the flow condition, but the direction of dot movement was random. In order to drive a maximal number of neurons sensitive to optic flow information, the expansion and contraction of optic flow changed several times per block. There were eight mini-blocks in total throughout each "flow" condition alternating between clockwise and counterclockwise flow during inward and outward contraction/expansion movement of dots, respectively. The direction of dot flow pattern changed between these eight mini-blocks every $500 \mathrm{~ms}$, synchronous with the appearance of a new set of dots (i.e., dot duration $=500 \mathrm{~ms}$ ). Participants were instructed to relax and maintain fixation in the center of the screen. Visual stimuli were presented with VisionEgg (Straw, 2008). Stimuli were projected from a rear-projection screen and reflected via a mirror that was attached to a 12-channel head coil.

\section{IMAGE ACOUISITION}

Participants were scanned using a Siemens Trio 3T scanner (Siemens Medical Systems, Erlangen, Germany) at the Athinoula A. Martinos Center for Biomedical Imaging. $\mathrm{T}_{1}$-weighted Magnetization Prepared-Rapid Acquisition Gradient Echo (MPRAGE) structural scans were acquired using generalized autocalibrating partially parallel acquisition (GRAPPA): repetition 
A

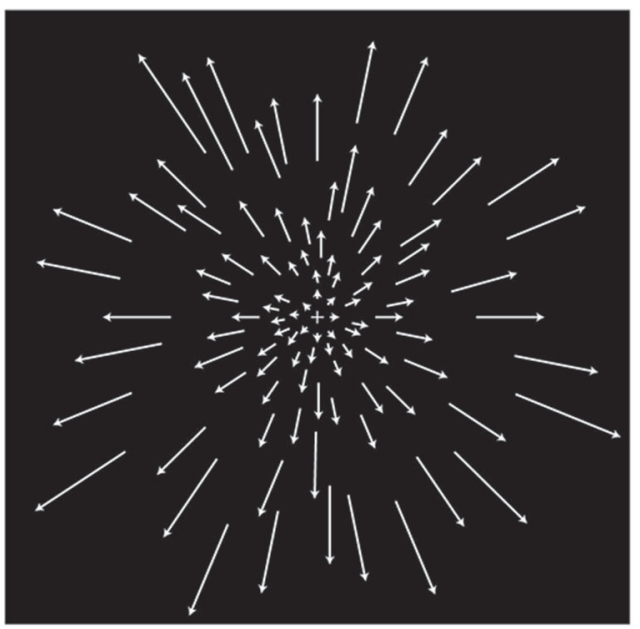

B

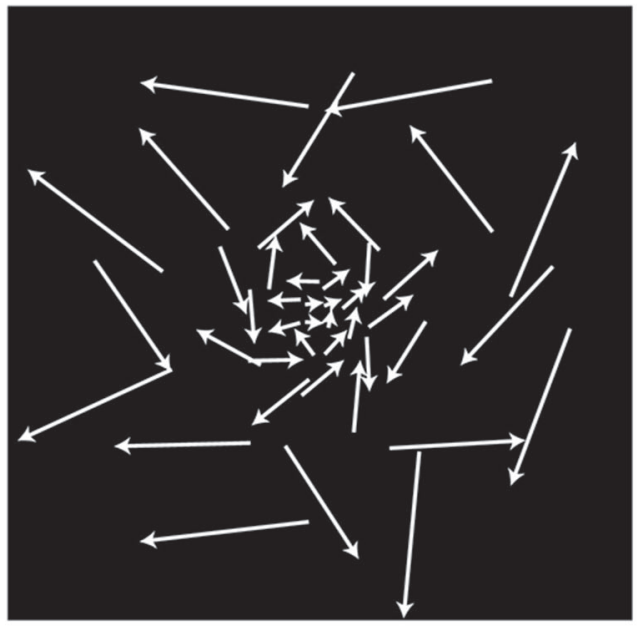

FIGURE 1 | Optic flow stimuli illustration. Optic flow motion stimuli (A) simulate forward and backward motion using dot fields that are expanding or contracting while rotating about a central focus. Random motion (B) simulates non-coherent motion using dots moving at the same speeds used in optic flow, but with random directions of movement. In the illustrations, the length of arrows corresponds with dot speed, indication that dot speed increases with distance away from the center time $=2530 \mathrm{~ms}$, echo time $=3.44 \mathrm{~ms}$, inversion time $=1100 \mathrm{~ms}$, flip angle $=7^{\circ}$, field of view $=256 \mathrm{~mm}$, slice thickness $=1 \mathrm{~mm}$, 176 sagittal slices (right to left). Blood oxygen level dependent (BOLD) fMRI data during presentation of visual stimuli were acquired using a-weighted gradient-echo echo-planar imaging (EPI) sequence: $\mathrm{TR}=2000 \mathrm{~ms}, \mathrm{TE}=30 \mathrm{~ms}, \mathrm{FA}=90^{\circ}$, FOV $=256 \mathrm{~mm}$, in-plane resolution $4 \times 4 \mathrm{~mm}^{2}$. Thirty-two axial (anterior to posterior) slices with a thickness of $4.0 \mathrm{~mm}$ were acquired, oriented parallel to the anterior-posterior commissural line. Six functional runs per scan session were acquired, each consisting of 128 whole-brain acquisitions collected immediately after acquisition of four "dummy" TRs for $\mathrm{T}_{1}$ stabilization.

\section{IMAGE PROCESSING AND ANALYSIS}

MP-RAGE images were processed using FreeSurfer (version 4.5.0). ${ }^{1}$ Standard preprocessing of structural volumes produced reconstructions that were used to determine if there were any areas of cortical atrophy. The FreeSurfer parcellations were also used to define individual calcarine sulcus labels from which to extract magnitude of activation during the task in each individual's primary visual cortex. This was done to ensure that activation in the primary visual cortex was comparable between $\mathrm{PD}$ and MC groups so that in the event that other cortical activation differences emerged, those results could be interpreted as a result of higher order visual processing. fMRI data were preprocessed using Statistical Parametric Mapping (SPM8; Wellcome Department of Cognitive Neurology, London, UK) for MatLab (The Mathworks, Inc, Natick, Massachusetts, USA). Functional data were re-oriented so that the origin was at the anterior commissure, and standard preprocessing was conducted including motion regression, segmentation, and spatial normalization

${ }^{1}$ http://surfer.nmr.mgh.harvard.edu into standard Montreal Neurological Institute (MNI) space using Diffeomorphic Anatomical Registration Through Exponentiated Lie algebra (DARTEL; Ashburner, 2007). Data were then smoothed using a $6 \mathrm{~mm}$ FWHM Gaussian kernel. Quality assurance by motion correction regression was conducted, and time points with $>2 \mathrm{~mm}$ of motion were modeled out of the analysis, which occurred for less than a third of all available timepoints for only three participants.

Trials were analyzed in a block design format. Conditions were classified as either flow or random (Figure 1). Following the methods used by Pitzalis et al. (2010), blocks were modeled as $16 \mathrm{~s}$ boxcars using the onset of each condition, and $T$ contrasts were constructed examining differences in fMRI activation during the flow compared to random condition within each individual to capture activation response to flow information that was not responsive to random motion. Analysis was based on a mixedeffects general linear model in SPM8.

\section{ANALYSIS MEASURES}

Functional whole brain activation maps of flow vs. random were generated in all participants together at a voxel activation threshold of $p<0.001$ cluster corrected to $p<0.01$ using an extent threshold of 46 contiguous resampled voxels calculated with the AFNI program 3dClustSimm. Next, we performed ROI analysis specific to the optic flow network. Peak activations in our five $a$ priori regions of interest (V6, V3A, MT+, CSv, and PIVC) were identified at the whole group level ( $\mathrm{PD}$ and $\mathrm{MC}$; Figure 2A). Coordinates of peaks are similar to previously published definitions of these regions (Tootell et al., 1997; Cardin and Smith, 2010; Pitzalis et al., 2010): MNI xyz: V6: RH[24 -76 32], LH[-20 -78 32]; V3A: RH[22 -84 20], LH[-22 -88 18]; MT+: RH[46 -58 2], LH[-40 -70 6]; PIVC: RH[44 -26 22], LH[-44 -32 20]; CSv: $\mathrm{RH}[14-2248], \mathrm{LH}[-12-2044] .5 \mathrm{~mm}$ radius spheres 


\section{A All Participants}
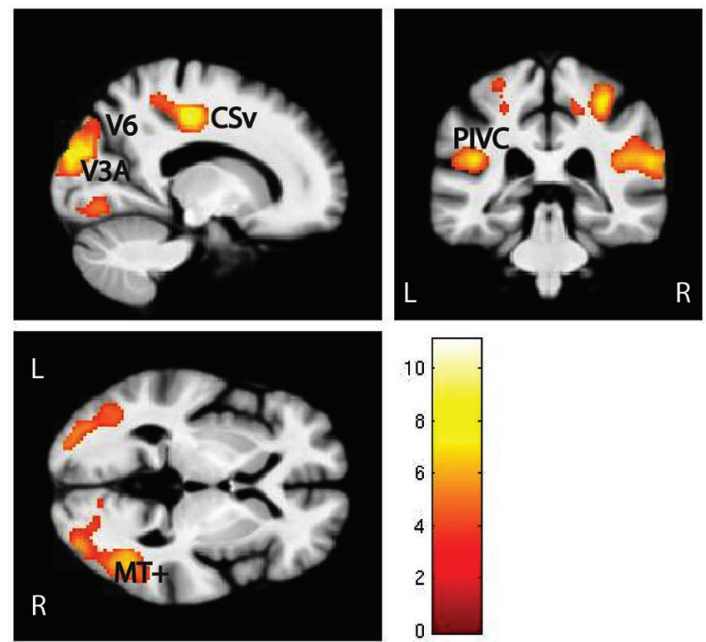

B $\quad M C>P D$
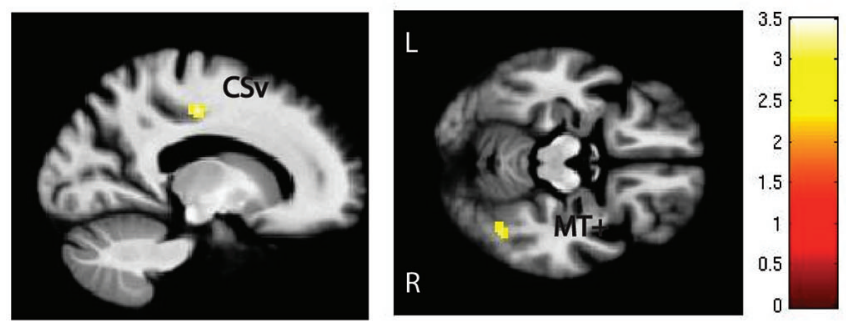

FIGURE 2 | Optic flow (flow > random) group activation. (A) Whole group activation in the optic flow network, including V6, V3A, MT+, PIVC, and CSv shown below, $p<0.001$, cluster corrected with a 46 voxel extent threshold to $p<0.01$, at MNI $x y z[-17-340]$. Scale bars represent the $t$-statistic. (B) Whole brain between group $t$-tests show

\section{$\mathrm{MC}>\mathrm{PD}$ in CSv and MT+}

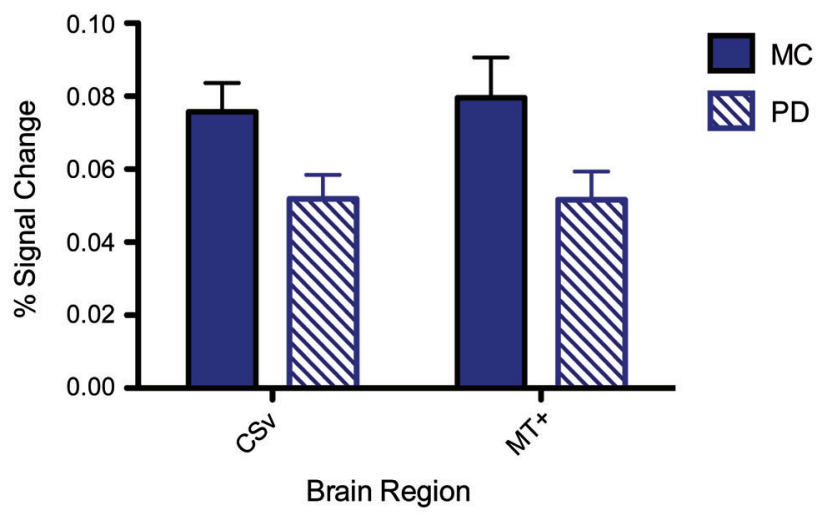

that CSv and MT+ are significantly more activated in MC than PD, $p<$ 0.01 cluster corrected with a 40 voxel extent threshold. Scale bars represent the $t$-statistic. (C) Results presented here are another visualization of magnitude of activation presented in (B), depicting higher activation in CSv and MT+ for MC than PD. around these peak activated voxels were constructed using the MarsBaR toolbox for SPM8. Magnitude of activation defined as percent signal change was then calculated from each of these ROIs. In order to test whether signal measured from the optic flow network ROIs during the flow vs. random contrast was significantly different for MC and PD, a Group $\times$ ROI analysis of variance was conducted on the extracted activation data, followed by post hoc two-sample $t$-tests. All measures of functional activation were averaged across hemispheres for every ROI to create a "bilateral" measure of activation. Next, bivariate correlations yielding Pearson's product-moment correlation coefficients were used to determine the association between activity in the ROIs and disease severity in the PD participants, as well as between ROI activity and behavioral performance on the coherent motion detection task in MC and PD separately.

\section{COHERENT MOTION BEHAVIORAL TASK}

A subset of $\mathrm{PD}(N=13)$ and $\mathrm{MC}(N=12)$ participants underwent a psychophysical experiment of coherent motion detection. The stimulus was a random dot pattern (RDP) containing signal and noise dots. The signal dots all moved either left or right on any given trial and the noise dots were assigned a random direction at the beginning of presentation and maintained that direction throughout the trial. All dots (200 dots, dot density $=$ 0.97 dots/deg) were white presented on a black background. The dot pattern was presented in a circular window subtending $16.2^{\circ}$ of visual angle with the signal and noise dots spatially interleaved. The stimulus was presented for $250 \mathrm{~ms}$ on a 21 " CRT monitor (Hewlett Packard P 1230) running at $120 \mathrm{~Hz}$ at $1024 \times 768$ resolution. Participants sat $61 \mathrm{~cm}$ from the screen. The task was to report the direction of the signal dots (left or right). The stimulus comprised 3, 6, 12, 24, 48, or $96 \%$ signal dots with the remainder being noise dots. The main outcome measure of the task was the coherent motion threshold. This was extracted by fitting a Weibull function (Britten et al., 1992) to the accuracy data across the six coherence levels for each participant. The threshold was defined as the minimum coherence level corresponding to $80 \%$ accuracy on the Weibull function (Norton et al., 2011).

\section{RESULTS}

PD IS ASSOCIATED WITH REDUCED ACTIVITY IN CSv AND MT+ DURING OPTIC FLOW PERCEPTION

ROI analysis focused on five cortical regions previously identified as key nodes of the optic flow network in healthy young 
adults (Cardin and Smith, 2010). These ROIs were defined from the significant activation patterns observed for all participants (Figure 2A). With respect to activation differences during the flow vs. random contrast between $\mathrm{MC}$ and $\mathrm{PD}$ groups, areas $\mathrm{CSv}$ and $\mathrm{MT}+$ showed lower activation at the whole brain level (Figures 2B,C). A Group $\times$ ROI ANOVA of percent signal change revealed a main effect of group $(F=4.23$, dof $=1, p=0.04)$, and a main effect of ROI $(F=3.12$, dof $=4, p=0.03)$. Post-hoc $t$-tests revealed significantly less activation in $\mathrm{PD}$ than $\mathrm{MC}$ specifically in $\operatorname{CSv}(t=2.34$, dof $=38, p=0.02)$ and MT $+(t=2.13$, dof $=38, p=$ $0.04)$. All other regions showed statistically comparable activation between groups: V6 $(t=1.46, d o f=38, p=0.16)$, V3A $(t=-0.26$, $d o f=38, p=0.79)$, PIVC $(t=1.29$, dof $=38, p=0.20)$. There were no group differences in V1 activity $(p>0.9)$.

\section{DISEASE SEVERITY IS RELATED TO ACTIVITY IN CSv}

Activation in CSv was negatively associated with disease severity within the PD group, as measured by the total UPDRS score $(r=-0.56, p=0.02)$, such that more severe disease was correlated with lower activation in this region (Figure 3 ). There were no significant associations between activation in the other optic flow network regions and disease severity.

\section{DISEASE SEVERITY IS RELATED TO COHERENT MOTION DETECTION THRESHOLDS}

In order to assess coherent motion processing behaviorally in $\mathrm{PD}$ and $\mathrm{MC}$, performance on the coherent motion task was analyzed for detection threshold at a level of $80 \%$ accuracy in each individual. There was no significant difference between MC and PD in detection thresholds $(p>0.4)$, suggesting that the PD group did not differ from MC in low-level motion processing. Within the PD group however, we found a positive association between disease severity and the coherent motion threshold $(r=0.69, p=$ 0.01 ), such that more progressed individuals with PD required higher dot coherency to successfully determine the direction of motion. This suggests that increased severity of PD is associated with impaired low-level motion perception. Coherent motion detection thresholds in the PD group did not correlate with activation in any regions of the optic flow network.

\section{DISCUSSION}

We examined the functional neural correlates of optic flow processing in PD in light of existing behavioral evidence suggesting that disruption in optic flow processing may underlie visuospatial deficits observed behaviorally (Davidsdottir et al., 2008; Young et al., 2010). In healthy young adults, visual cortical areas V6, V3A, and MT+ have been characterized as extracting coherent motion cues selective for self-motion (Cardin and Smith, 2010, 2011; Pitzalis et al., 2010). In addition to these visual motion areas, two regions thought to process and integrate visuo-vestibular input have also been identified as responsive to optic flow stimuli: PIVC and CSv (Wall and Smith, 2008; Cardin and Smith, 2010). In the present study, we established that healthy older adults and individuals with PD also process optic flow in this network of regions similar to what has been observed in young adults. Importantly, we found that individuals with PD showed changes in activation patterns compared to the healthy aging group within

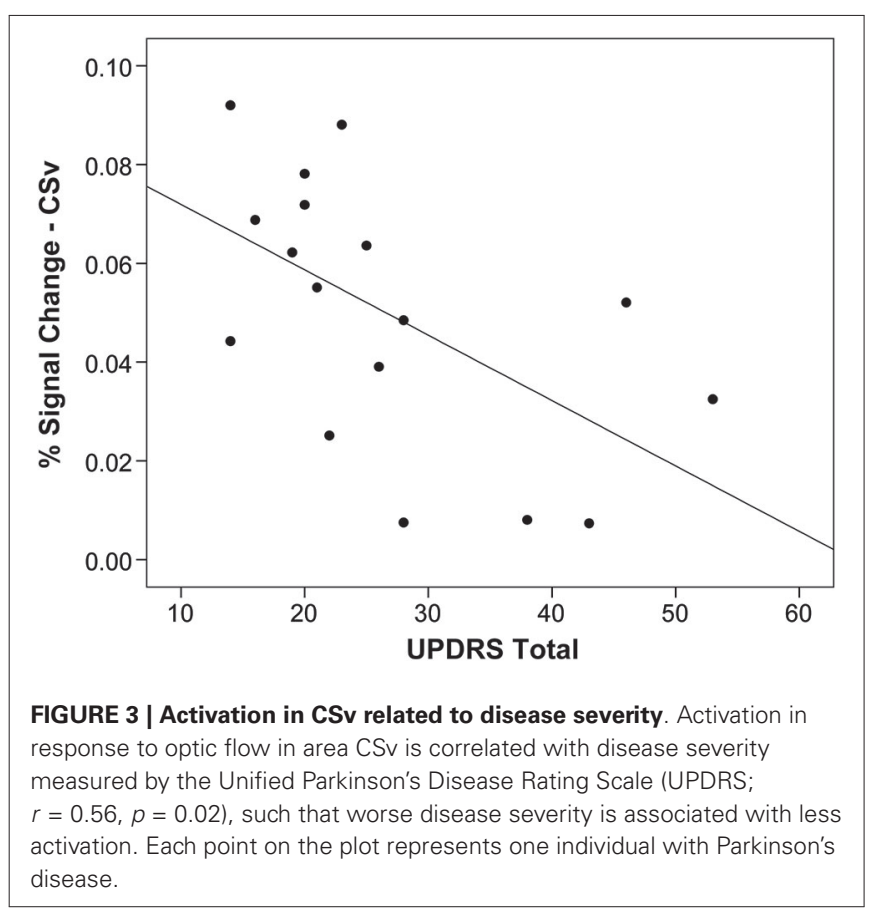

the optic flow network overall, and specifically, demonstrated less activity within visual motion area $\mathrm{MT}+$ and the visuo-vestibular region CSv in response to perception of optic flow.

Additionally, within the PD participants, disease severity was correlated with activity in CSv during the optic flow task, further suggesting that this region is targeted by $\mathrm{PD}$ pathology. These findings provide new evidence delineating functional neural correlates of disrupted optic flow processing that have been demonstrated behaviorally in PD (Davidsdottir et al., 2008). Further, activity in V1 during this task, together with normal visual acuity, confirmed intact lower-level vision, suggesting that the difference between groups in optic flow processing derives specifically from compromised functioning in regions of the optic flow network. Although the PD participants in this study did not differ behaviorally from MC in the ability to detect direction of coherent motion, success on this task was related to disease severity scores in $\mathrm{PD}$, indicating that within this group of mild-to-moderate PD, those who demonstrated difficulty detecting direction of coherent motion were also those with more severe disease-related impairment. Though it is important to consider the effect of levodopa medication status on these findings, we established that measurements of Levodopa Equivalent Dosage in our PD participants were not significantly related to disease severity or activation in CSv, rendering the drug effect unlikely to fully account for our observations.

Motion information feeds forward from lower-level visual motion areas (V1, MT+, V3A, V6) to more anterior regions of this optic flow network (PIVC and CSv), where vestibular information about body position in space is processed to create an integrated sense of optic flow perception relative to the self (Browning et al., 2009). PIVC and CSv receive inputs from V6 and MT+ (Cardin and Smith, 2010) and activate in 
response to stimuli consistent with coherent egomotion (Wall and Smith, 2008; Cardin and Smith, 2010). CSv has previously been described as a region that integrates visual input with vestibular signals, and has been implicated in the visual and postural orientation of oneself in the environment (Dean and Platt, 2006; Vogt et al., 2006; Cardin and Smith, 2010; Smith et al., 2012). Individuals with PD have demonstrated difficulty with proprioceptive orientation (Jacobs and Horak, 2006; Vaugoyeau and Azulay, 2010), consistent with our finding of reduced functional activation in $\mathrm{CSv}$ in response to optic flow stimuli.

These findings suggest that PD pathology influences optic flow processing even in the mild to moderate stages of the disease. An important caveat is that our PD participants were studied "ON" medication, and it may be of interest to examine participants off medication in future studies. The observed reduction in functional activity during optic flow processing, particularly in CSv, may underlie proprioceptive integration deficits that have been identified as giving rise to later postural instability (Vaugoyeau and Azulay, 2010) and visuospatial dysfunction (Amick et al., 2006; Stepkina et al., 2010; Poletti et al., 2012). Because changes at the neural level often precede striking behavior changes, we postulate that as the disease progresses, the failure to integrate egomotion visual cues with vestibular input in PD leads to a decline in visuoperceptual cognitive function. We suggest that the observed reduction in activation in $\mathrm{MT}+$ and $\mathrm{CSv}$ may precede early signs of visuospatial cognitive decline and postural instability as the disease progresses.

\section{ACKNOWLEDGMENTS}

We thank all of the individuals who participated in this study. Our recruitment efforts were supported, with our gratitude, by Marie Saint-Hilaire, MD, and Cathi Thomas RN, MSN, of Boston Medical Center Neurology Associates, and by Boston area Parkinson disease support groups. We thank Chelsea Toner, M.A., and Laura Pistorino, B.A., for recruitment support. The authors declare no competing financial interests. This work was supported by the National Institute of Health R01 NS067128. Scanning was carried out at the Athinoula A. Martinos Center for Biomedical Imaging at the Massachusetts General Hospital, which receives funding from P41EB015896, a Biotechnology Resource Grant supported by the National Institute of Biomedical Imaging and Bioengineering (NIBIB), National Institutes of Health. This work also involved the use of instrumentation supported by the NIH Shared Instrumentation Grant Program and/or High-End Instrumentation Grant Program; specifically, grant number(s) S10RR022976 and S10RR019933. We would also like to acknowledge NEI grant number NIH R01EY022229 for supporting the efforts of co-authors Maya L. Rosen and Deepti Putcha.

\section{REFERENCES}

Amick, M. M., Schendan, H. E., Ganis, G., and Cronin-Golomb, A. (2006). Frontostriatal circuits are necessary for visuomotor transformation: mental rotation in Parkinson's disease. Neuropsychologia 44, 339-349. doi: 10.1016/j. neuropsychologia.2005.06.002

Armstrong, R. A. (2011). Visual symptoms in Parkinson's disease. Parkinsons Dis. 2011:908306. doi: 10.4061/2011/908306

Ashburner, J. (2007). A fast diffeomorphic image registration algorithm. Neuroimage 38, 95-113. doi: 10.1016/j.neuroimage.2007.07.007
Britten, K. H., Shadlen, M. N., Newsome, W. T., and Movshon, J. A. (1992). The analysis of visual motion: a comparison of neuronal and psychophysical performance. J. Neurosci. 12, 4745-4765.

Browning, N. A., Grossberg, S., and Mingolla, E. (2009). A neural model of how the brain computes heading from optic flow in realistic scenes. Cogn. Psychol. 59, 320-356. doi: 10.1016/j.cogpsych.2009.07.002

Cardin, V., and Smith, A. T. (2010). Sensitivity of human visual and vestibular cortical regions to egomotion-compatible visual stimulation. Cereb. Cortex 20, 1964-1973. doi: 10.1093/cercor/bhp268

Cardin, V., and Smith, A. T. (2011). Sensitivity of human visual cortical area V6 to stereoscopic depth gradients associated with self-motion. J. Neurophysiol. 106, 1240-1249. doi: 10.1152/jn.01120.2010

Chou, Y. H., Wagenaar, R. C., Saltzman, E., Giphart, J. E., Young, D., Davidsdottir, R., et al. (2009). Effects of optic flow speed and lateral flow asymmetry on locomotion in younger and older adults: a virtual reality study. J. Gerontol. B Psychol. Sci. Soc. Sci. 64, 222-231. doi: 10.1093/geronb/gbp003

Davidsdottir, S., Wagenaar, R., Young, D., and Cronin-Golomb, A. (2008). Impact of optic flow perception and egocentric coordinates on veering in Parkinson's disease. Brain 131, 2882-2893. doi: 10.1093/brain/awn237

Dean, H. L., and Platt, M. L. (2006). Allocentric spatial referencing of neuronal activity in macaque posterior cingulate cortex. J. Neurosci. 26, 1117-1127. doi: 10.1523/jneurosci.2497-05.2006

Duffy, C. J. (2009). Visual motion processing in aging and Alzheimer's disease: neuronal mechanisms and behavior from monkeys to man. Ann. N Y Acad. Sci. 1170, 736-744. doi: 10.1111/j.1749-6632.2009.04021.x

Durant, S., and Zanker, J. M. (2012). Variation in the local motion statistics of real-life optic flow scenes. Neural Comput. 24, 1781-1805. doi: 10. 1162/neco_a_00294

Jacobs, J. V., and Horak, F. B. (2006). Abnormal proprioceptive-motor integration contributes to hypometric postural responses of subjects with Parkinson's disease. Neuroscience 141, 999-1009. doi: 10.1016/j.neuroscience.2006. 04.014

Movement Disorder Society Task Force on Rating Scales for Parkinson's Disease. (2003). The Unified Parkinson's Disease Rating Scale (UPDRS): status and recommendations. Mov. Disord. 18, 738-750. doi: 10.1002/mds.10473

Norton, D. J., Mcbain, R. K., Ongur, D., and Chen, Y. (2011). Perceptual training strongly improves visual motion perception in schizophrenia. Brain Cogn. 77, 248-256. doi: 10.1016/j.bandc.2011.08.003

Pitzalis, S., Galletti, C., Huang, R. S., Patria, F., Committeri, G., Galati, G., et al. (2006). Wide-field retinotopy defines human cortical visual area v6. J. Neurosci. 26, 7962-7973. doi: 10.1523/jneurosci.0178-06.2006

Pitzalis, S., Sereno, M. I., Committeri, G., Fattori, P., Galati, G., Patria, F., et al. (2010). Human v6: the medial motion area. Cereb. Cortex 20, 411-424. doi: 10. 1093/cercor/bhp112

Poletti, M., De Rosa, A., and Bonuccelli, U. (2012). Affective symptoms and cognitive functions in Parkinson's disease. J. Neurol. Sci. 317, 97-102. doi: 10. 1016/j.jns.2012.02.022

Seiffert, A. E., Somers, D. C., Dale, A. M., and Tootell, R. B. (2003). Functional MRI studies of human visual motion perception: texture, luminance, attention and after-effects. Cereb. Cortex 13, 340-349. doi: 10.1093/cercor/13. 4.340

Smith, A. T., Wall, M. B., and Thilo, K. V. (2012). Vestibular inputs to human motion-sensitive visual cortex. Cereb. Cortex 22, 1068-1077. doi: 10. 1093/cercor/bhr179

Stepkina, D. A., Zakharov, V. V., and Yakhno, N. N. (2010). Cognitive impairments in progression of Parkinson's disease. Neurosci. Behav. Physiol. 40, 61-67. doi: 10. 1007/s11055-009-9223-6

Straw, A. D. (2008). Vision egg: an open-source library for realtime visual stimulus generation. Front. Neuroinform. 2:4. doi: 10.3389/neuro.11.004. 2008

Tinaz, S., Courtney, M. G., and Stern, C. E. (2011). Focal cortical and subcortical atrophy in early Parkinson's disease. Mov. Disord. 26, 436-441. doi: 10. $1002 / \mathrm{mds} .23453$

Tomlinson, C. L., Stowe, R., Patel, S., Rick, C., Gray, R., and Clarke, C. E. (2010). Systematic review of levodopa dose equivalency reporting in Parkinson's disease. Mov. Disord. 25, 2649-2653. doi: 10.1002/mds.23429

Tootell, R. B., Mendola, J. D., Hadjikhani, N. K., Ledden, P. J., Liu, A. K., Reppas, J. B., et al. (1997). Functional analysis of V3A and related areas in human visual cortex. J. Neurosci. 17, 7060-7078. 
Vaugoyeau, M., and Azulay, J. P. (2010). Role of sensory information in the control of postural orientation in Parkinson's disease. J. Neurol. Sci. 289, 66-68. doi: 10. 1016/j.jns.2009.08.019

Vogt, B. A., Vogt, L., and Laureys, S. (2006). Cytology and functionally correlated circuits of human posterior cingulate areas. Neuroimage 29, 452-466. doi: 10. 1016/j.neuroimage.2005.07.048

Wall, M. B., and Smith, A. T. (2008). The representation of egomotion in the human brain. Curr. Biol. 18, 191-194. doi: 10.1016/j.cub.2007.12.053

Warren, W. H. Jr., Kay, B. A., Zosh, W. D., Duchon, A. P., and Sahuc, S. (2001). Optic flow is used to control human walking. Nat. Neurosci. 4, 213-216. doi: 10. $1038 / 84054$

Young, D. E., Wagenaar, R. C., Lin, C. C., Chou, Y. H., Davidsdottir, S., Saltzman, E., et al. (2010). Visuospatial perception and navigation in Parkinson's disease. Vision Res. 50, 2495-2504. doi: 10.1016/j.visres.2010. 08.029
Conflict of Interest Statement: The authors declare that the research was conducted in the absence of any commercial or financial relationships that could be construed as a potential conflict of interest.

Received: 30 May 2014; accepted: 24 June 2014; published online: 08 July 2014. Citation: Putcha D, Ross RS, Rosen ML, Norton DJ, Cronin-Golomb A, Somers DC and Stern CE (2014) Functional correlates of optic flow motion processing in Parkinson's disease. Front. Integr. Neurosci. 8:57. doi: 10.3389/fnint.2014.00057 This article was submitted to the journal Frontiers in Integrative Neuroscience. Copyright (c) 2014 Putcha, Ross, Rosen, Norton, Cronin-Golomb, Somers and Stern. This is an open-access article distributed under the terms of the Creative Commons Attribution License (CC BY). The use, distribution or reproduction in other forums is permitted, provided the original author(s) or licensor are credited and that the original publication in this journal is cited, in accordance with accepted academic practice. No use, distribution or reproduction is permitted which does not comply with these terms. 